\title{
Propagation of ultra-short solitons in stochastic Maxwell's equations
}

\author{
Levent Kurt \\ Division of Mathematics and Natural Sciences \\ Queens College \\ City University of New York \\ Queens, $N Y$ \\ Tobias Schäfer \\ Department of Mathematics \\ College of Staten Island \\ City University of New York \\ Staten Island, NY*
}

\begin{abstract}
We study the propagation of ultra-short short solitons in a cubic nonlinear medium modeled by nonlinear Maxwell's equations with stochastic variations of media. We consider three cases: variations of (a) the dispersion, (b) the phase velocity, (c) the nonlinear coefficient. Using a modified multi-scale expansion for stochastic systems, we derive new stochastic generalizations of the short pulse equation that approximate the solutions of stochastic nonlinear Maxwell's equations. Numerical simulations show that soliton solutions of the short pulse equation propagate stably in stochastic nonlinear Maxwell's equations and that the generalized stochastic short pulse equations approximate the solutions to the stochastic Maxwell's equations over the distances under consideration. This holds for both a pathwise comparison of the stochastic equations as well as for a comparison of the resulting probability densities.
\end{abstract}

* tobias@math.csi.cuny.edu 


\section{INTRODUCTION}

Many integrable partial differential equations arise as asymptotic expansions of nonlinear wave equations. In the particular context of ultra-short pulses propagating in cubic nonlinear media, the basic model equation is given by Maxwell's wave equation with dispersion and nonlinearity coming from the material's response to the excitation by the electric field [1]. When the pulse width is large in comparison to the carrier wavelength, an asymptotic expansion of the nonlinear wave equation yields the cubic nonlinear Schrödinger equation (NLSE) [2]. The NLSE is integrable [3] and has bright soliton solutions that have been studied intensively over the last four decades, not only because of their mathematical beauty, but also because of a variety of possible applications in modern optical technology [4]. Recent experimental progress in the field of ultra-short pulses [5] has led to increased interest in models that go beyond the NLSE description: As the length of the pulse shortens, the basic assumption of scale separation between envelope and carrier wave is not satisfied anymore [6] and additional correction terms have to be added [7]. On the other hand, it is possible to introduce a different scaling [8] in the asymptotic analysis of the nonlinear wave equation that is appropriate for ultra-short pulses. In this way, it is possible to derive a different approximation of the solution of Maxwell's equation, the short pulse equation (SPE) [9]. Although derived under entirely different assumptions in comparison to the NLSE, the SPE is integrable [10] as well and was shown to possess bright solitons that may be as short as three cycles [11]. Consequently, over the last years, the mathematical structure of the SPE was subject to intensive research [12-17].

When a solitary wave solution is taken as the initial condition in the SPE, it persists for arbitrary propagation distances. As initial condition of the original nonlinear wave equation, however, it is expected to change its shape due to the fact that the SPE is only an approximation to the nonlinear wave equation. If $\epsilon$ is the expansion factor in the multiple-scale asymptotic analysis, we would expect to see a noticeable deviation only after propagation distances of the order of $1 / \epsilon$ [18]. We show at the end of the following section numerically by propagating the soliton to a distance of order $1 / \epsilon^{2}$ that this change happens indeed very slowly, such that we can say that the SPE solitons persist in Maxwell's equations. The slow change of the soliton solution is governed by higher-order effects that appear as addi-

tional terms in the multi-scale expansion and that can be studied systematically [19]. In the 
present paper, since we are focusing on the leading order impact of stochastic perturbations of the parameters in the equations, we neglect these higher-order deterministic effects. Incorporating deterministic higher-order effects in the presence of random perturbations lies beyond the scope of this paper and will be studied in the future.

Such random fluctuations are widely present in nature and models in which stochasticity is taken into account are more realistic than deterministic models. One important question that arises in the context of integrable systems is whether soliton solutions persist when perturbed stochastically: NLSE solitons broaden under the influence of stochastic variations of the dispersion coefficient [20] . For the NLSE case, however, this broadening is sufficiently slow such that the soliton still persists over long distances, provided that the stochastic perturbations are sufficiently small. One result of this paper is that SPE solitons show similar behavior.

When studying the impact of stochastic variations of the media, one faces the problem of coarse-graining noise, namely the question of how microscopic variations of the parameters in the nonlinear wave equation relate to variations of the parameters in the SPE or the NLSE. In section III, based on previous work [21, 22], we show how this coarse-graining of noise can be done explicitly in the multi-scale expansion that leads from the nonlinear wave equation to the SPE: We introduce randomness in the linear dispersive part of the susceptibility of the media and obtain a stochastic dispersion coefficient in the SPE, yielding a stochastic generalization of the short pulse equation. Similarly, stochasticity can be introduced in the phase velocity and and in the nonlinearity. The resulting generalized stochastic short pulse equations constitute the main result of this work.

It remains to be shown that the solutions to the stochastic short pulse equations stay close to the solutions of the original stochastic nonlinear wave equation. Even in the deterministic context this is very challenging and, in fact, this problem has only be solved recently [23]. Therefore, in section IV we present a numerical comparison of the solutions to the nonlinear wave equation with randomness and the corresponding stochastic short pulse equations. We show that, over the distances under consideration, SPE solitons also persist in the stochastic short pulse equations and in the stochastic nonlinear wave equation. Moreover, extensive numerical simulations show that the SPE model can be used to predict the likelihood of large deviations from the deterministic solution when we compare particular stochastic paths and as well as the corresponding probability distributions. 


\section{ULTRA-SHORT SOLITONS IN DETERMINISTIC MAXWELL'S EQUATIONS}

In this section we briefly review the setting of the short pulse equation and its derivation from Maxwell's equations. We first consider the following deterministic one-dimensional wave equation describing pulse propagation in bulk silica [9]

$$
u_{x x}-u_{t t}=\chi_{0} u+\chi_{3}\left(u^{3}\right)_{t t}
$$

Here, $x$ is our evolution variable and we assume that we are given initial conditions $u(x=0, t)$ and $u_{x}(x=0, t)$. In this section we assume the coefficients $\chi_{0}$ and $\chi_{3}$ to be fixed, in the next section we will modify the problem by allowing the coefficients $\chi_{0}$ and $\chi_{3}$ to vary stochastically. Above equation can be directly derived from Maxwell's equations under the assumption that the response of the medium is instantaneous, otherwise a retarded response of the material will lead to convolution integrals in the polarization which will yield a nonlocal wave equation [24. We also assume that the Fourier transform $\hat{u}$ is zero for very low wavelengths. This accounts for the fact that such waves cannot propagate in the optical medium.

As for many wave equations, we can use asymptotic expansions in order to construct approximate solutions to (1). The short pulse equation (SPE) can be derived from (1) using a multiple-scale expansion [9] or an equivalent method like the renormalization group method [25]: A multi-scale expansion of the form

$$
u(x, t)=\epsilon A_{0}\left(\phi, x_{1}, x_{2}, \ldots\right)+\epsilon^{2} A_{1}\left(\phi, x_{1}, x_{2}, \ldots\right)+\ldots
$$

with

$$
\phi=\frac{t-x}{\epsilon}, \quad x_{n}=\epsilon^{n} x
$$

yields as leading order

$$
-2 \partial_{\phi} \partial_{x_{1}} A_{0}=\chi_{0} A_{0}+\chi_{3} \partial_{\phi}^{2} A_{0}^{3}
$$

This form of the short pulse equation can be transformed to the standard form

$$
U_{X T}=U+\frac{1}{6} U_{X X}^{3}
$$

through the transformation given by

$$
A_{0}\left(\phi, x_{1}\right)=\sqrt{\frac{\chi_{0}}{6 \chi_{3}}} U(X, T), \quad X=-\phi, \quad T=\frac{\chi_{0}}{2} x_{1} .
$$


Note that we change here from the physical variables $\left(x_{1}, \phi\right)$ (which are rescaled and shifted with respect to the variables $(x, t)$ of the nonlinear wave equation) to the mathematical variables $(T, X)$. In all simulation results later, we will always use the physical variables $(x, t)$ of the original nonlinear wave equation.

To our knowledge, it is unknown whether the original wave equation (1) is integrable. Sakovich and Sakovich, however, have shown that the SPE (5) is integrable and, moreover, admits one-soliton solutions [11] that can be written as

$$
\begin{aligned}
U & =4 m n \frac{m \sin \psi \sinh \phi+n \cos \psi \cosh \phi}{m^{2} \sin ^{2} \psi+n^{2} \cosh ^{2} \phi} \\
X & =Y+2 m n \frac{m \sin 2 \psi-n \sinh 2 \phi}{m^{2} \sin ^{2} \psi+n^{2} \cosh ^{2} \phi}
\end{aligned}
$$

with

$$
\phi=m(Y+T), \quad \psi=n(Y-T), \quad n=\sqrt{\left(1-m^{2}\right)} .
$$

This family of solutions depends on the parameter $m$, and the condition for these solitary wave solutions being non-singular was found to be

$$
m<m_{c r}=\sin \frac{\pi}{8} \approx 0.383
$$

The parameter $m$ determines the shortness of the pulse. As $m$ reaches its critical value, the shortest pulse of this family we can construct is approximately a three cycle pulse. When $m$ is small (for instance, $m=0.01$ ), solitary wave solution of the SPE can be approximated by

$$
U \approx 4 m \cos (X-T) \operatorname{sech}(m(X+T))
$$

such that the pulse is similar to a bright soliton solution of the cubic nonlinear Schrödinger equation. In the present work, we focus on the behavior of ultra-short solutions. In our simulations, we take $m=0.3$.

With these soliton solutions of the SPE at hand, the question arises whether they also show stable propagation when taken as initial conditions in the original problem (1). First, it was shown for the linear case $\left(\chi_{3}=0\right)$ that the solution of the SPE stays close to the solution of the original problem [25]. For the nonlinear case, the resulting proof is much more difficult and a solution was found only recently [23].

Figure 1 shows a numerical solution of the nonlinear wave equation (1) with the SPE soliton solution taken as initial conditions. In the simulation, we chose the parameter $m$ 
to be 0.3 and $\epsilon=0.2$. This snapshot of the soliton profile is compared to the analytical solution of the SPE given by (7). The soliton shows stable propagation in the nonlinear wave equation - note that the evolution distance in $x$ is $25.625 \approx \mathcal{O}\left(1 / \epsilon^{2}\right)$, which is significantly further than we would expect for a leading order multi-scale expansion. The reason for this can be found when considering addtional higher-order terms in the multi-scale expansion [19]. In this work, however, we neglect such high-order contributions.

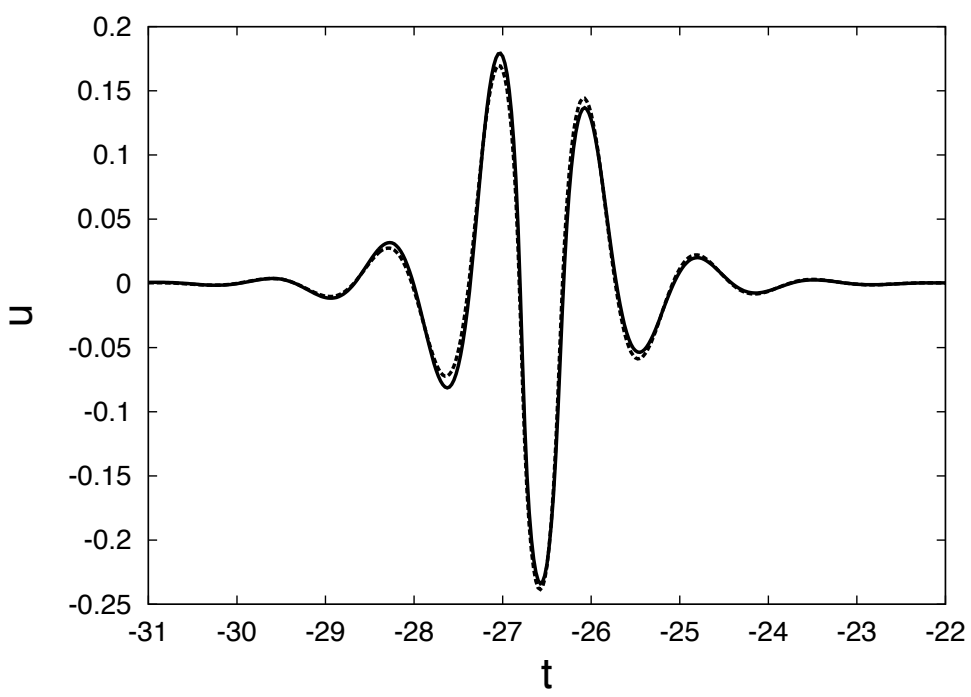

FIG. 1. Profile of the SPE soliton in the nonlinear wave equation (1). The solid line shows the snapshot of the soliton solution as a result of its propagation in Maxwell's nonlinear wave equation. The dashed line is the corresponding solution to the short pulse equation with the same initial condition. The small deviations are due to higher-order deterministic corrections.

In general, we expect the difference between the solution $u$ of the nonlinear wave equation and the soliton $u_{s}$ to grow with the propagation distance $x$. We can quantify this growth by a numerical comparison. In figure 2 we plot the evolution of several norms of $u-u_{s}$, in particular the $L^{1}, L^{2}$ and $L^{\infty}$-norms defined by

$$
\|f\|_{L^{k}}=\left(\int|f(t)|^{k} d t\right)^{1 / k}, \quad\|f\|_{L^{\infty}}=\max (|f(t)|) .
$$

Whereas the $L^{1}$ and the $L^{2}$-norms display monotonic growth, the $L^{\infty}$-norm shows oscillations that are due to the fact that this norm is very sensitive to shifts in time as it compares the solutions pointwise. 


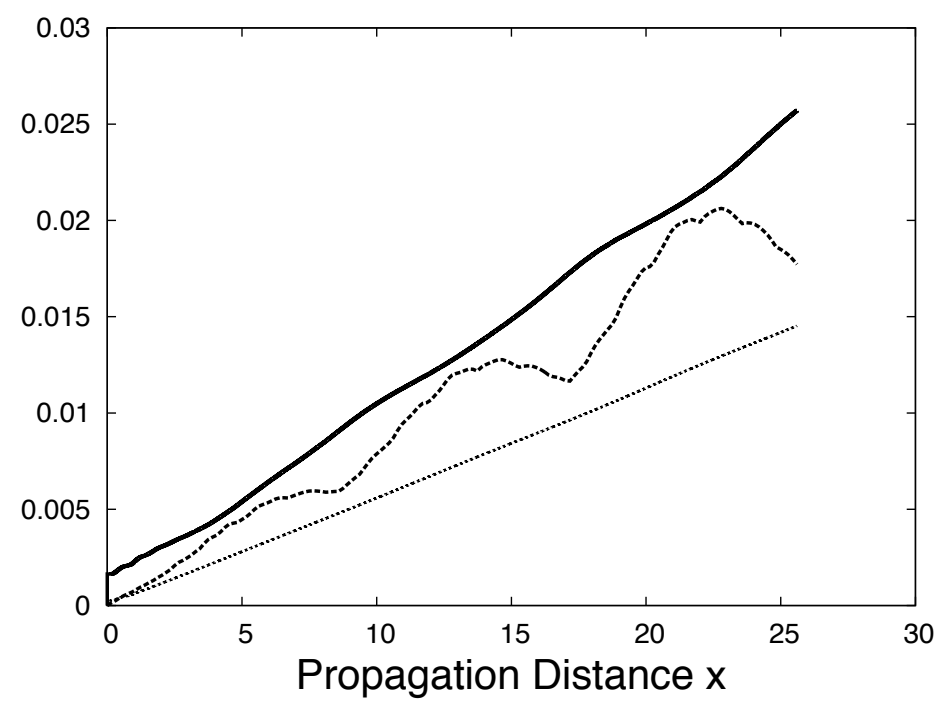

FIG. 2. Evolution of the deviations of the SPE soliton when propagating in the nonlinear wave equation (1). The solid line shows the evolution of the $L^{1}$-norm given by $\left\|u-u_{s}\right\|_{L^{1}}$, the dashed line the evolution of the $L^{\infty}$-norm, and the dotted line the evolution of the $L^{2}$-norm.

\section{THE STOCHASTIC SHORT PULSE EQUATION}

Optical soliton propagation in fibers in the presence of a stochastic perturbation has been studied intensively in the context of the NLSE model [20]. The sources of randomness in optical fibers may vary. For example, the phase of the wave may fluctuate due to the presence of the randomness. One major source of medium-related stochastic phase fluctuations are the inhomogeneities in a fiber's core or the fluctuations in the linear refractive index of the core. Stimulated Brillouin scattering, stimulated Raman scattering, and inhomogeneities of the medium are the possible sources of the phase fluctuations [26, 27]. The stochasticity may come from the nonlinearity of the medium as well. The dynamical effect of the noise added by the stochastic nature of the nonlinearity is much smaller than the noise due to the inhomogeneities [28]. Nevertheless, there may be other sources of randomness playing a role, and they may originate from the other parts of the system such as the inherent power fluctuation in lasers used as input pumps. Apart from these, quantum phase fluctuations are also sources of phase noise in optical fibers although they are practically negligible [7]. In many applications, Langevin noise is used to study the fluctuations in the system [1]. 
In the present work, we do not model the precise microscopic origins of the randomness, but rather assume that they lead to small stochastic variations of the coefficients of the equations. This approach is common, one particular case studied in the past is the NLSE with a linear multiplicative stochastic term corresponding to stochastic variations of the dispersion. In analogy, it is natural to ask how the ultra-short solitons of the deterministic SPE propagate in a stochastic environment.

Therefore, the simplest corresponding stochastic variations can be introduced to the wave equation (1) by making the coefficient $\chi_{0}$ representing the linear susceptibility stochastic. Setting

$$
\chi_{0}=\bar{\chi}_{0}+\chi_{\text {rand }}(x), \quad \chi_{\text {rand }}(x)=\nu \xi(x),
$$

leads to the stochastic wave equation such that

$$
u_{x x}-u_{t t}=\left[\bar{\chi}_{0}+\nu \xi(x)\right] u+\chi_{3}\left(u^{3}\right)_{t t}
$$

where $\xi$ represents white noise, hence $\left\langle\xi(x) \xi\left(x^{\prime}\right)\right\rangle=\delta\left(x-x^{\prime}\right)$, and the coefficient $\nu$ measures the strength of the noise. Although mathematically convenient, we note that this is only an approximation, as in reality, the noise will be short-correlated rather than delta-correlated. In the numerical simulations we also will assume that the coefficient of the dispersive term $\chi_{0}$ will always be larger than zero which correctly represents the underlying physical system.

In order to derive a stochastic generalization of the short pulse equation, we introduce the same multi-scale expansion as in the deterministic case of the form

$$
u(x, t)=\epsilon M_{0}\left(\phi, x_{0}, x_{1}, x_{2}, \ldots\right)+\epsilon^{2} M_{1}\left(\phi, x_{0}, x_{1}, x_{2}, \ldots\right)+\ldots
$$

with again rescaled variables

$$
\phi=\frac{t-x}{\epsilon}, \quad x_{n}=\epsilon^{n} x
$$

As in the deterministic case, we solve now the equation order by order. As terms of the order $1 / \epsilon$ cancel, we obtain at $\mathcal{O}(1)$ the equation $M_{0 \phi x_{0}}=0$ such that the leading order $M_{0}$ is independent of the fast variable $x_{0}$. The equation for $M_{1}$ is given by a combination of terms and can be written as:

$$
-2\left(M_{1}\right)_{x_{0} \phi}=2\left(M_{0}\right)_{x_{1} \phi}+\left(\bar{\chi}_{0}+\nu \xi(x)\right) M_{0}+\chi_{3}\left(M_{0}^{3}\right)_{\phi \phi}
$$

In the deterministic case, if $\nu=0$, the solvability condition of this equation yields the short pulse equation, and therefore the entire r.h.s. of 16 will be zero. For the stochastic case, 
the standard multi-scale argument has to be slightly modified due to the presence of the noise term. If we integrate (16) with respect to the fast variable $x_{0}$ from $x_{0}=0$ to $x_{0}=1$, the noise term $\xi(x)$ yields a random number $\zeta_{0}$ given by $\zeta_{0}=\int_{0}^{1} \xi(x) d x$. As $\xi$ is white noise, $\zeta_{0}$ is normally distributed with mean zero and variance one. On the slow scale $x_{1}$, the points 0 and 1 correspond to 0 and $\epsilon$ and $\zeta_{0}$ represents the cumulative microscopic noise for this interval. For the next interval $[1,2)$ on the fast scale (corresponding to $[\epsilon, 2 \epsilon$ ) on the slow scale) we can repeat the process and obtain $\zeta_{1}=\int_{1}^{2} \xi(x) d x$. In this way we obtain a sequence of independent normally distributed random numbers $\left(\zeta_{k}\right)=\left(\zeta_{0}, \zeta_{1}, \ldots\right)$. On the slow scale, the random number $\zeta_{k}$ characterizes the noise in the $k$-th interval $[k \epsilon,(k+1) \epsilon)$. Hence, we can view the sequence of random numbers $\left(\zeta_{k}\right)$ on the slow scale as a discretization of a white noise process $\Xi=\Xi\left(x_{1}\right)$ with $\left\langle\Xi\left(x_{1}\right) \Xi\left(x_{1}^{\prime}\right)\right\rangle=\epsilon \delta\left(x_{1}-x_{1}^{\prime}\right)$. In this way we obtain the stochastic short pulse equation with coarse-grained noise as

$$
-2\left(M_{0}\right)_{x_{1} \phi}=\left(\bar{\chi}_{0}+\nu \Xi\left(x_{1}\right)\right) M_{0}+\chi_{3}\left(M_{0}^{3}\right)_{\phi \phi}
$$

One important difference between the deterministic and the stochastic case is that, if we introduce (17) back in (16), we obtain a dependence of $M_{1}$ on the fast variable $x_{0}$ that is not present in the deterministic case. This is especially important when considering the next order $\mathcal{O}\left(\epsilon^{2}\right)$ in the asymptotic expansion. In this work, however, we only consider terms up to the order $\mathcal{O}(\epsilon)$.

Note that we obtain the strength of the slow-scale noise $\Xi$ explicitly and that both the microscopic strength of the noise (given by $\nu$ ) and the expansion coefficient $\epsilon$ influence the strength of the coarse-grained noise. The reason for the fact that the coarse-graining of the noise takes a rather simple form lies in the fact that the randomness is introduced in the linear dispersive term and that the leading order evolution is trivial as $M_{0}$ is independent of $x_{0}$. In cases where the leading order solution is more complicated, its structure will influence the strength of the coarse-grained noise [22].

Aside from fluctuations in the dispersion, other parameters might vary stochastically as well. In deriving the short pulse equation from Maxwell's equations, an approximate fit of the linear susceptibility of the form

$$
\hat{\chi}^{(1)}(\lambda) \approx \hat{\chi}_{0}^{(1)}-\hat{\chi}_{2}^{(1)} \lambda^{2}
$$

in Fourier domain [9, 29] was made. This susceptibility approximation is found by fitting the experimental data for light propagation in silica fibers in the infrared regime. The first 
stochastic generalization of the SPE treated above is derived by considering the fluctuations in the $\lambda$-dependent term. However, one can argue that the fluctuations may come from the $\hat{\chi}_{0}^{(1)}$ term in 18 rather than the $\hat{\chi}_{2}^{(1)}$ term, corresponding to stochastic variations of the phase velocity. This treatment will lead to a different stochastic wave equation:

$$
u_{x x}-[1+\nu \xi(x)] u_{t t}=\chi_{0} u+\chi_{3}\left(u^{3}\right)_{t t}
$$

As before, a multiple scale expansion, see (2) and (3), leads to a different form of the stochastic SPE. As the calculations are similar to the previous case of random dispersion, we just give the result:

$$
-2\left(M_{0}\right)_{x_{1} \phi}=\chi_{0} M_{0}+\chi_{3}\left(M_{0}^{3}\right)_{\phi \phi}+\left(\frac{\nu}{\epsilon^{2}} \Xi\left(x_{1}\right)\right)\left(M_{0}\right)_{\phi \phi}
$$

Finally, we will introduce the noise in the nonlinearity as well, which will result in a stochastic wave equation in the form of

$$
u_{x x}-u_{t t}=\chi_{0} u+\left[\bar{\chi}_{3}+\nu \xi(x)\right]\left(u^{3}\right)_{t t}
$$

The corresponding stochastic SPE takes the form

$$
-2\left(M_{0}\right)_{x_{1} \phi}=\chi_{0} M_{0}+\left(\bar{\chi}_{3}+\nu \Xi\left(x_{1}\right)\right)\left(M_{0}^{3}\right)_{\phi \phi}
$$

If one sets $\nu=0$ in any of these stochastic generalizations of the SPE, one will obtain the deterministic short pulse equation (4).

\section{NUMERICAL COMPARISON}

\section{A. Pathwise comparison}

Again, we expect (17) to be a decent approximation of the nonlinear wave equation (1) with a stochastic linear susceptibility given by 12 for propagation distances that are of the order of at least $\mathcal{O}(1 / \epsilon)$. Since we obtained a very good agreement up to distances of $\mathcal{O}\left(1 / \epsilon^{2}\right)$ in the deterministic case, we will consider this as our maximum propagation distance for the stochastic case as well.

In order to evaluate the quality of the approximation numerically, we can look at a pathwise comparison. From the coarse-graining procedure described above, we obtain for any 
particular realization $\xi_{p}$ of the micro-scale noise $\xi$ on the fast scale a macroscopic noise $\Xi_{p}$ on the slow scale. Then, we can compare the solution of the stochastic SPE (17) with the solution of the nonlinear wave equation for this particular realization of the noise. Again we choose to look at the differences of the solution of the stochastic PDEs and the deterministic soliton. For the simulation of the nonlinear wave equation, we subtract the error that exists in the deterministic system, presented in figure 2. As a result, we obtain two stochastic processes - one given by the difference of the solution of the stochastic SPE and the deterministic soliton and the other given by the difference of the solution of the stochastic nonlinear wave equation and the deterministic solution to the wave equation (with the SPE soliton as initial condition).

The result of the corresponding numerical simulations is shown in figure 3 and shows excellent agreement of the approximation and the solution of the original equation.

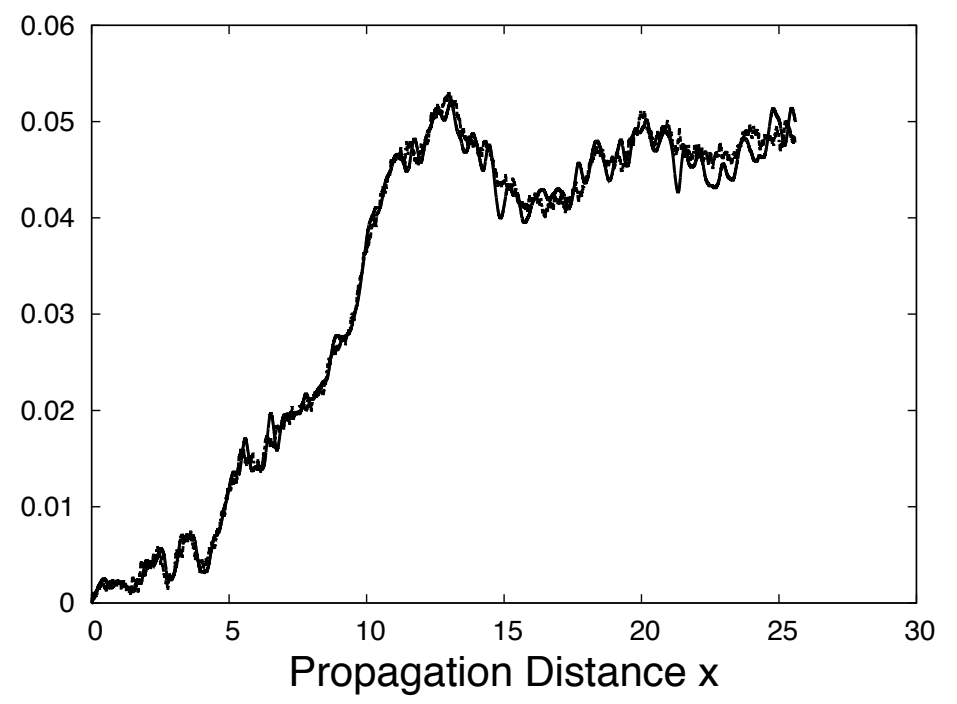

FIG. 3. Stochastic dispersion: Evolution of the stochastic difference of the SPE soliton and the solution of the nonlinear wave equation (1). The solid line shows the evolution of the $L^{\infty}$-norm of the difference generated by the solution of the stochastic nonlinear wave equation and the dashed line shows the corresponding difference for the corresponding evolution in the stochastic short pulse equation. The strength parameter $\nu$ of the microscopic noise was 0.125 .

The next figure 4 shows the same comparison for the case of randomness in the phase 
velocity. In this case, we compare the solutions to (20) to the solutions of (1) for the case of random variations of $\hat{\chi}_{0}^{(1)}$.

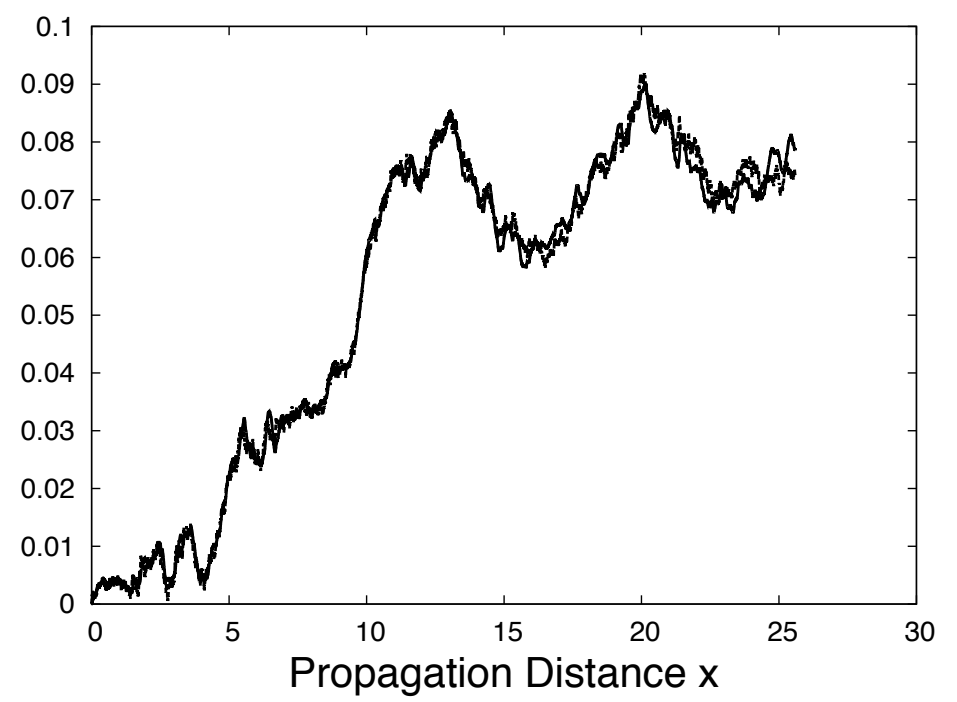

FIG. 4. Stochastic phase velocity: Evolution of the stochastic difference of the SPE soliton and the solution of the nonlinear wave equation (1) for the case of random phase velocity. The solid line shows the evolution of the $L^{\infty}$-norm of the difference generated by the solution of the stochastic nonlinear wave equation and the dashed line shows the corresponding difference for the corresponding evolution in the stochastic short pulse equation. The strength parameter $\nu$ of the microscopic noise was 0.005 . Recall that $\epsilon=0.2$ such that the ratio $\nu / \epsilon^{2}$ is still fairly small.

The third case is the comparison for the case of random fluctuation of the nonlinear coefficient. Figure 5 compares the solution of the stochastic short pulse equation with the solution to stochastic Maxwell's equations.

Hence, for all three cases, we have numerical evidence that the coarse-grained stochastic generalizations of the SPE capture correctly the impact of the noise on the solitons when propagating in the stochastic nonlinear wave equation. 


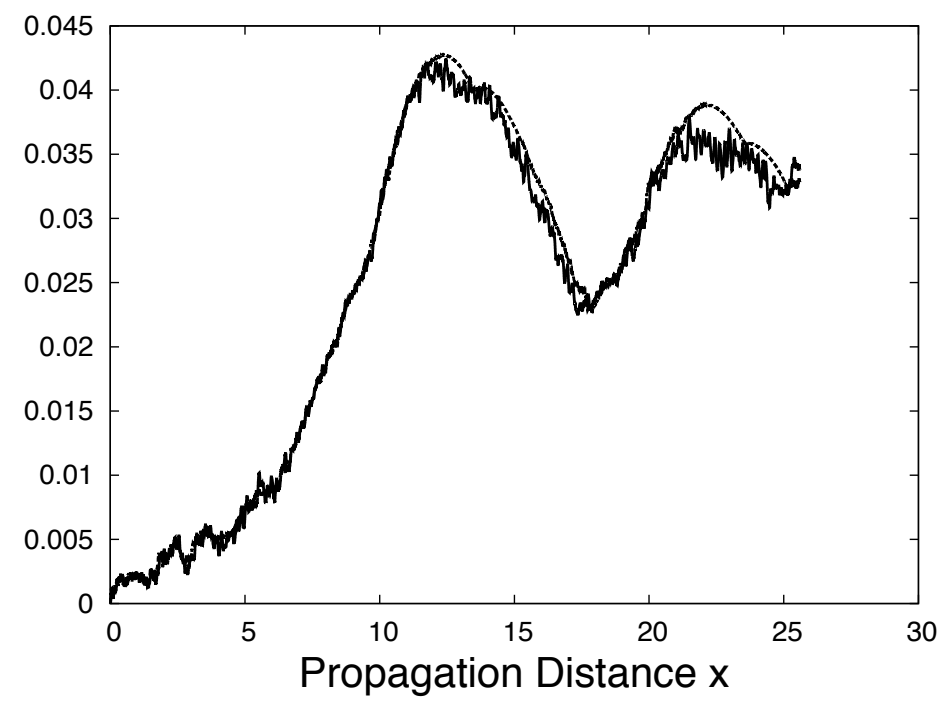

FIG. 5. Stochastic phase velocity: Evolution of the stochastic difference of the SPE soliton and the solution of the nonlinear wave equation (11) for the case of random phase velocity. The solid line shows the evolution of the $L^{\infty}$-norm of the difference generated by the solution of the stochastic nonlinear wave equation and the dashed line shows the corresponding difference for the corresponding evolution in the stochastic short pulse equation. The strength parameter $\nu$ of the microscopic noise was 0.05 .

\section{B. Probability distribution}

This pathwise correspondence indicates that we can also expect a decent agreement in the prediction of the related probability distributions. To check this, we can plot the histograms

of the deviations of the solutions to the stochastic PDEs from the deterministic evolution of the SPE solitons. The following figure 6 was obtained by creating a probability distributions from 10000 realizations of the stochastic short pulse equation and stochastic Maxwell's equation for the case of stochastic variations of the dispersion.

Although we see a small difference of the SPE and stochastic Maxwell's equations for small deviations, we obtain overall an excellent agreement between the approximation given by the stochastic short pulse equation and the stochastic nonlinear wave equation. In particular, the shape of the probability distribution is correctly predicted by the SPE approximation and large deviations from the mean are correctly accounted for as well. 


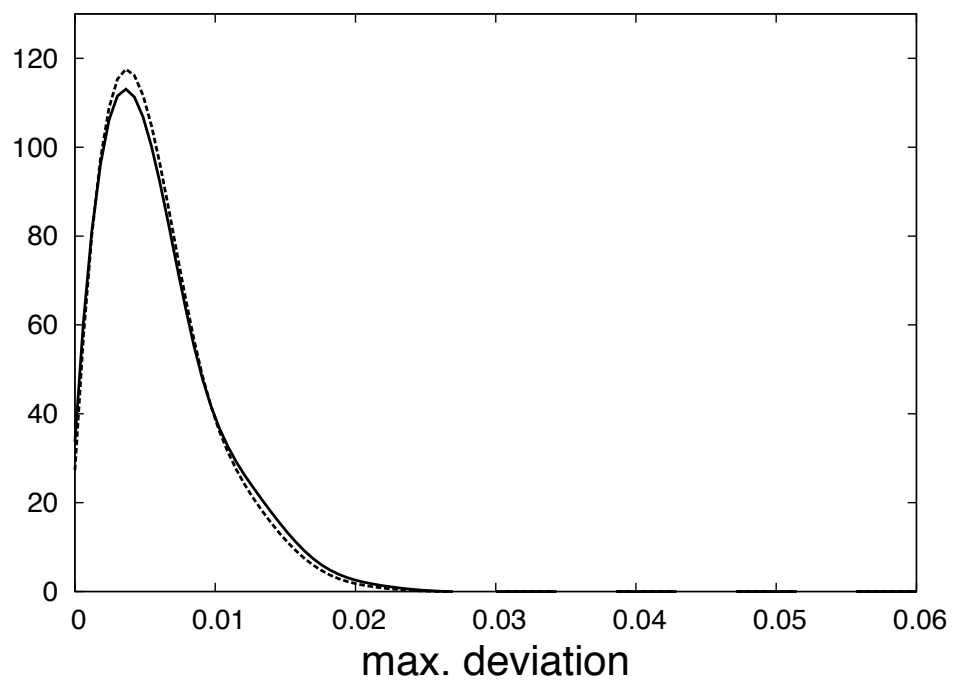

FIG. 6. Probability distributions of the stochastic difference of the SPE soliton and the solution of the nonlinear wave equation (1) at the propagation distance $x=25.625$. The $x$-axis shows the $L^{\infty}$-norm of the difference generated by the solution of the stochastic PDEs in comparison to the deterministic solution (solid line: Maxwell, dashes: SPE). In this way, the solution of the nonlinear wave equation has been corrected for the deterministic error. The strength of the noise was $\nu=0.05$.

\section{CONCLUSION}

In this paper we have derived stochastic generalizations of the short pulse equation and shown explicitly how the noise processes governing the parameters of the SPE can be constructed from the noise present in the underlying nonlinear wave equation. Moreover, we have shown that SPE solitons persist in the nonlinear wave equation with stochastic variations present and that they exhibit only small changes over the propagation distances under consideration. The changes arising from the random part of the susceptibility can be correctly characterized by the stochastic short pulse equation. 


\section{ACKNOWLEDGMENTS}

This work was partially supported by National Science Foundation through the grants DMS-0807396 and DMS-1108780 and through the grant of the PSC-CUNY research foundation PSCREG-39-709. The authors gratefully acknowledge the support of the CUNY High Performance Computing Facility and the Center for Interdisciplinary Applied Mathematics and Computational Sciences. The authors are grateful to D. Trubatch for many valuable discussions.

[1] R. W. Boyd. Nonlinear Optics. Academic Press, Boston, 1992.

[2] A. C. Newell and J. V. Moloney. Nonlinear Optics. Addison-Wesley, Redwood City, CA, 1992.

[3] V. E. Zakharov and A. B. Shabat. Exact theory of two-dimensional self-focussing and onedimensional self-modulation of waves in nonlinear media. Zh. Ekcp. Teor. Fiz, 61:118-134, 1971.

[4] A. Hasegawa and Yuki Kodama. Solitions in optical communications. Clarendon Press, Oxford, 1995.

[5] N. Karasawa, S. Nakamura, N. Nakagawa, M. Shibata, R. Morita, H. Shigekawa, and M. Yamashita. Comparision between theory and experiment of nonlinear propagation for a-few-cycle and ultrabroadband optical pulses in a fused-silica fiber. IEEE J. Quant. Elect., 37:398-404, 2001.

[6] J. E. Rothenberg. Space-time focusing: breakdown of the slowly varying envelope approximation in the self-focusing of femtosecond pulses. Opt. Lett., 17:1340-1342, 1992.

[7] G. P. Agrawal. Nonlinear Fiber Optics. Academic Press, Burlington, MA, 2007.

[8] D. Alterman and J. Rauch. Diffractive short pulse asymptotics for nonlinear wave equations. Phys. Lett. A, 264:390-395, 2000.

[9] T. Schäfer and C. E. Wayne. Propagation of ultra-short optical pulses in cubic nonlinear media. Physica D, 196:90-105, 2004.

[10] A. Sakovich and S. Sakovich. The short pulse equation is integrable. J. Phys. Soc. Jpn., 74:239-241, 2005. 
[11] A. Sakovich and S. Sakovich. Solitary wave solutions of the short pulse equation. J. Phys. A: Math. Gen., 39:L361-L367, 2006.

[12] J. C. Brunelli. The short pulse hierarchy. J. Math. Phys., 46:123507, 2005.

[13] A. Sakovich and S. Sakovich. On transformations of the rabelo equations. SIGMA, 3:086, 2007.

[14] Y. Matsuno. Multiloop soliton and multibreather solutions of the short pulse model equation. Journal of the Physical Society of Japan, 76(8):084003-084008, 2007.

[15] K. K. Victor, B. B. Thomas, and T. C. Kofane. On exact solutions of the Schäfer-Wayne short pulse equation: WKI eigenvalue problem. J. Phys. A: Math. Theor., 40:5585-5596, 2007.

[16] V. Manukian, N. Costanzino, C. K. R. T. Jones, and B. Sandstede. Existence of MultiPulses of the Regularized Short-Pulse and Ostrovsky Equations. Journal of Dynamics and Differential Equations, 21:607-622, 2009.

[17] D. Pelinovsky and A. Sakovich. Global well-posedness of the short-pulse and sine-Gordon equations in energy space. Commun. Part. Diff. Eq. 35, pages 613-629, 2010.

[18] M. H. Holmes. Introduction to Perturbation Methods. Springer, New York, 1995.

[19] L. Kurt, Y. Chung, and T. Schäfer. Higher-order corrections to the short-pulse equation. arXiv:1205.1343 [nlin.SI], 2012.

[20] F. Kh. Abdullaev, J. C. Bronski, and G. Papanicolaou. Soliton perturbations and the random Kepler problem. Physica D, 135:369-386, 2000.

[21] T. Schäfer, R. O. Moore, and C. K. R. T. Jones. Pulse propagation in media with deterministic and random dispersion variations. Optics Communications, 214:353-362, 2002.

[22] T. Schäfer and R. O. Moore. A path integral method for coarse-graining noise in stochastic differential equations with multiple time scales. Physica D, 240:89-97, 2011.

[23] D. Pelinovsky and G. Schneider. Rigorous justification of the short-pulse equation. arXiv:1108.5970 [math-ph], 2011.

[24] Y. Chung and T. Schäfer. Stabilization of ultra-short pulses in cubic nonlinear media. Phys. Lett. A, 361:63-69, 2007.

[25] Y. Chung, C.K.R.T. Jones, T. Schäfer, and C. E. Wayne. Ultra-short pulses in linear and nonlinear media. Nonlinearity, 18:1351-1374, 2005.

[26] D.L. Hart, A.F. Judy, R. Roy, and J.W. Beletic. Dynamical evolution of multiple four-wavemixing processes in an optical fiber. Phys. Rev. E, 57:4757-4774, 1998. 
[27] C. Sulem and P.L. Sulem. The Nonlinear Schrödinger Equation Self-Focusing and Wave Collapse. Springer-Verlag, New York, 1999.

[28] F. Kh. Abdullaev, J.H. Hensen, S. Bischoff, and M.P. Sorensen. Propagation and interaction of optical solitons in random media. J. Opt. Soc Am. B., 15:2424-2432, 1998.

[29] L. Kurt. Modeling of Ultra-Short Solitons in Deterministic and Stochastic Nonlinear Cubic Media. Ph.D. thesis, The Graduate Center and University Center, The City University of New York, 2011. 